\title{
Comunicación de una experiencia clínica con autodaño desde el enfoque estratégico de terapia breve
}

\author{
Communication of the clinical experience with self \\ injury from the brief strategic approach therapy
}

Carolina Calderón P. ${ }^{1}$

\begin{abstract}
Resumen
El objetivo de este artículo es dar cuenta de un proceso terapéutico de enfoque estratégico empleado en un caso único de Self Injury Behavior. Las estrategias centrales de la intervención fueron la reformulación y prescripción de comportamiento, encontrando, en el enfoque estratégico, una alternativa para el tratamiento de esta clase de síntomas considerados de difícil tratamiento.

Palabras clave: Self Injury Behavior, Enfoque Estratégico, Redefinición, Prescripción de Comportamiento.
\end{abstract}

\begin{abstract}
The objective of this article is to explain the therapeutic process based on a strategic approach employed in a unique case of Self Injury Behavior. The redefinition and prescription of the behavior were the central strategies of intervention, finding, in the strategic approach, an intervention alternative to these kinds symptoms, which are considered both difficult and serious.

Key words: Self Injury Behavior, Strategic Approach, Redefinition, Prescription Behavior.
\end{abstract}

Psicóloga. Magister en Psicología Clínica. Bogotá, Colombia. clonyrimbaud@hotmail.com 


\section{Introducción}

El autodaño o Self Injury Behavior (SIB) se define como un síntoma en el que se presentan acciones frecuentes que dañan de manera directa el tejido fino del cuerpo sin la intención de suicidarse (Whitlock y Knox, 2007). El corte en la piel parece ser el tipo más común de auto - lesión, pero otras formas incluyen quemarse, rasguñarse, golpearse e interferir con el proceso de curación de una herida (Klonsky, 2007).

Dentro de la nosología psiquiátrica, el SIB es incluido como un síntoma o elemento diagnóstico del desorden de la personalidad limítrofe y de otros cuadros psiquiátricos incluyendo: depresión, desórdenes de ansiedad, abuso de sustancias, trastornos alimenticios, estrés post traumático y esquizofrenia (American Psychiatric Association, 2000).

Las lesiones corporales se presentan de forma moderada sobre la superficie corporal, como los cortes, el tallado o la quema de la piel, distinguiéndose de otras formas de autodaño más severo como enucleación (sacarse los ojos), castración y amputación de partes del cuerpo, en su mayoría asociadas a episodios psicóticos (Claes y Vandereycken, 2007).

Siguiendo a los autores, el SIB se clasifica teniendo en cuenta los siguientes aspectos: 1 . El tipo de acción que se usa para dañarse (cortarse, morderse, la inserción, quemarse, ingestión o inhalación y golpearse); 2. La localización corporal del daño (cabeza, torso superior, torso inferior o extremidades); 3. La frecuencia con que la persona se autolesiona; 4. El grado de daño de la herida sobre la piel y la gravedad de la misma.

El SIB se caracteriza por la relación directa o la inmediatez del acto, es decir, el vínculo entre el comportamiento y la consecuencia física, una herida, es inmediata e inequívoca. En otras formas de hacerse daño (los desórdenes de alimentación, abuso de sustancias o fingirse enfermo) este vínculo es indirecto, remoto y dudoso. Cuando la persona comunica la existencia del SIB, por lo general, admite haberse lesionado ella misma y es consciente del origen psicológico del daño (Klonsky, 2007).

La conducta de autodaño principalmente se lleva a cabo de manera privada y, por lo general, no se busca atención médica, lo que hace 
difícil conocer el nivel de prevalencia del SIB dentro de la población. Cabe señalar que, hasta el momento, la mayoría de los estudios del SIB se han realizado con pacientes en tratamiento y diagnosticados dentro de cuadros psiquiátricos severos (Purington y Whitlock, 2004). La excepción es un estudio realizado en Estados Unidos (Whitlock, Eckenrode y Silverman, 2006), que toma como muestra estudiantes universitarios. Aquí se observa un aumento en la prevalencia del SIB, presentándose con mayor frecuencia en mujeres jóvenes, entre 17 y 22 años, con historial de abuso sexual, trastornos alimenticios o con cuestionamiento de su identidad sexual. El $75 \%$ de la muestra acepta haberse autodañado y sólo el 3,5\% reconoce haber buscado ayuda médica. El estudio citado concluye que son pocas las investigaciones orientadas hacia la detección, intervención y estrategias de tratamiento que se llevan a cabo en el SIB.

Cabe señalar que la mayoría de las investigaciones se centra en la clasificación del síntoma y los criterios diagnósticos, e indican que, en general, el tratamiento del SIB ha estado en manos de la psiquiatría (incluye el uso de fármacos del tipo antidepresivos y antipsicóticos) y de la terapia conductual, donde se busca sustituir la conducta problema por una más adaptativa, por medio de la extinción de la conducta problema y el reforzamiento de la nueva conducta (Claes y Vandereycken, 2007).

Finalmente, se reconoce que el SIB es actualmente un problema psiquiátrico crónico y frecuente dentro de los hospitales mentales y la consulta psicológica, presentando un desafío para los terapeutas, teniendo en cuenta la gravedad de la conducta que, en muchas ocasiones, pone en peligro la vida del cliente (Graff y Mallin, 1967).

Considerando lo anterior, el objetivo de este artículo es comunicar un caso clínico exitoso donde se presentó el SIB y fue aplicado el enfoque estratégico de terapia breve (MRI), mostrando este enfoque como una alternativa de tratamiento útil en el manejo de este síntoma. A continuación se presentarán los aspectos teóricos y prácticos clave que se tuvieron en cuenta en el proceso terapéutico que se quiere comunicar.

Los principios fundamentales del enfoque estratégico de terapia breve ${ }^{2}$

Dura menos de 20 sesiones. 
surgieron en la década del '70 en el Mental Research Institute de Palo Alto, California, Estados Unidos. Este modelo es parte de los modelos sistémicos de intervención a los cuales también pertenecen el modelo estructural y modelo de Milán. Desde esta perspectiva, se entiende que los clientes o consultantes hacen parte de un sistema (familia, escuela, sociedad, etc.) que está conformado por otros miembros. Es en la constante interacción donde los miembros construyen pautas o maneras de relación que se mantienen y que comunican la forma de sentir, pensar y actuar de ese sistema y de sus miembros ante las diversas experiencias humanas. Por consiguiente, es en las relaciones donde se busca intervenir, siendo éste el foco desde donde se construye el problema o motivo de consulta, el proceso y las intervenciones terapéuticas.

\section{Intervención Enfoque Estratégico}

Este modelo de intervención se basa en una postura epistemológica constructivista, la cual reconoce y valida la capacidad del hombre de construir su realidad y de participar de ésta constantemente por medio de la experiencia. Como lo afirman Neimeyer y Mahoney (1995): "el constructivismo es una perspectiva epistemológica basada en la afirmación de que los seres humanos crean activamente las realidades a las que responden" (p. 89).

El enfoque aplicado se centra en la extinción de los síntomas y en la resolución del problema o de los problemas presentados por el paciente o pacientes, por medio de una reestructuración o modificación del modo de percibir la realidad y de las reacciones comportamentales derivadas del paciente o pacientes (Nardone y Watzlawick, 1992).

Desde la perspectiva del modelo estratégico del MRI, el problema está determinado por el mecanismo de acciones y retroacciones desencadenado y mantenido por las "soluciones ensayadas" que el paciente o los pacientes han utilizado para hacer frente a los trastornos o síntomas actuales. Estas acciones y retroacciones generan pautas y circuitos de relación mantenidos por el sistema consultante (Casabianca y Hirsch, 1994). 
En pocas palabras, el problema o motivo de consulta se construye teniendo en cuenta los miembros involucrados dentro del sistema que consulta, las interacciones que se dan entre ellos y los comportamientos o estrategias que se han utilizado para hacer frente a la dificultad. Estas estrategias buscan, por lo general, eliminar o controlar el síntoma o la conducta problema pero, por el contrario, la conducta problema se mantiene durante un tiempo, hasta que es leída por uno o varios miembros del sistema como inmodificable, insoportable o de gran gravedad.

Cabe señalar que las pautas interaccionales se clasifican en simétricas y complementarias. En las pautas simétricas los miembros del sistema tienden, en las respuestas relacionales, a igualar su conducta. Por ejemplo, A grita y B le grita más duro, dándose la escalada simétrica. En las pautas complementarias las conductas de los miembros del sistema tienden a ser diferentes o antagónicas entre sí; por ejemplo, A acosa y B se retira, con secuencias sucesivas: mientras más A acosa, mayor es el retiro de $\mathrm{B}$ (Watzlawick, Beavin y Jackson, 1997).

$\mathrm{Al}$ construir el problema terapéutico y las diversas estrategias hay que orientarse hacia objetivos aparentemente mínimos o meta minima. Si el terapeuta identifica bien la secuencia o secuencias de acciones y la pauta o pautas que mantienen y favorecen la existencia y función del síntoma o síntomas dentro del sistema, un pequeńo cambio detalladamente estudiado puede ser suficiente para producir un gran efecto (Nardone y Watzlawick, 1992).

El problema o motivo de consulta suele implicar los siguientes rasgos: 1. La preocupación manifestada por un cliente acerca de una conducta propia o de otra persona con la que se halla significativamente comprometido. 2. Dicha conducta es considerada, implícita o explícitamente, desviada de la norma, como real o potencialmente molesta o dañosa, para quien la emite o para los demás. 3. Se han hecho esfuerzos para modificar esa conducta, pero no han tenido éxito (Fisch, Segal y Weakland, 1988).

Existen diferentes estrategias terapéuticas que permiten alterar el circuito que mantiene el problema. Se mencionan aquellas que posibilitan 
comprender mejor la intervención usada en el presente caso. La primera es la reformulación, que consiste en poner un suceso dentro de contextos diferentes y mirarlo desde perspectivas diversas. Si se cambia el punto de observación, cambia también la manera de ver la realidad (Feixas y Villegas, 2004).

La reformulación además puede tener diversos niveles de complejidad, que van desde simples redefiniciones cognoscitivas de una idea o de un comportamiento, hasta complicadas reestructuraciones paradójicas, pasando por el uso de metáforas y sugestiones evocativas (Nardone y Watzlawick, 1992).

En segundo lugar están las prescripciones de comportamiento, que son tareas que se dan a los consultantes y construyen con base en su cotidianeidad y experiencia concreta. Éstas pueden realizarse dentro o fuera de la sesión. Entre las prescripciones se encuentran las de tipo directo e indirecto. Las de tipo directo son indicaciones claras de acciones que hay que hacer, y las de tipo indirecto se dan cuando se pide hacer algo con el fin de producir una cosa distinta a lo que se ha declarado o prescrito (Nardone y Watzlawick, 1992).

Cabe mencionar que dentro del proceso terapéutico se reconocen tres niveles de intervención. El nivel de la construcción o significado, el nivel de la acción y el nivel de emoción. El objetivo es intervenir para perturbarlos y de esta manera generar cambios en los circuitos de explicaciones de la experiencia y de la acción. Esto es lo que Procter (1987) denomina como posición, y que define como la postura integrada que asume cada miembro del sistema, suponiendo la inclusión de los niveles ya mencionados.

En resumen, desde esta perspectiva los problemas o síntomas que aquejan a los clientes, en este caso el SIB, surgen en interacción dentro de un contexto determinado, y dentro de este contexto se construye un significado que explica, para cada uno de los involucrados, la conducta problemática. El significado o explicación que se le da al SIB dentro del sistema familiar genera una acción o acciones, las cuales se presentan con el fin de resolver el problema o eliminar el síntoma. 
La conducta de SIB se mantiene por los diferentes intentos de solución que, en lugar de resolver el problema, lo refuerzan, intensificando la sensación, en muchos clientes, de no tener control sobre lo que ocurre y calificarlo de inmodificable, como en el caso de la experiencia clínica que a continuación se presenta.

\section{Método}

\section{Presentación del Caso Clínico}

El caso se registró haciendo uso de la ficha clínica propuesta por Casabianca y Hirsch (1994): "la que permite comparar el trabajo de un terapeuta con otros, comparar distintos momentos de un mismo proceso y guiar la dirección del trabajo terapéutico" (p. 19). Las fichas permiten registrar desde la primera entrevista lo que ocurre en cada sesión, teniendo en cuenta el motivo de consulta, las soluciones intentadas y la totalidad del proceso terapéutico. Esta herramienta permite comunicar posteriormente la experiencia clínica que se registró.

En el presente artículo se comparte el motivo de consulta construido con el sistema consultante, se presentan los circuitos de acción involucrados, el objetivo terapéutico, las estrategias aplicadas y finalmente la evolución del caso, aspectos que dan cuenta de la aplicación del modelo. Simultáneamente se refieren de manera textual algunas narraciones de los miembros de la familia que evidencian su postura y aspectos de su experiencia.

Los nombres reales de los clientes fueron cambiados para resguardar su anonimato. El caso clínico se nombró usando la metáfora Escrito en el cuerpo. Se realizó un total de 13 sesiones (una sesión semanal). La mayoría fueron individuales, con la paciente identificada y, otras, con miembros de su familia (principalmente la madre). Se llevó a cabo en el Centro de Atención Psicológica Sistémica de Santiago de Chile, CAPSIS, contando con supervisión clínica. 


\section{Antecedentes del problema clínico}

La paciente, Catalina, es una adolescente de 14 años que vive en Santiago, estudiante de enseñanza media en un establecimiento educacional público. Es la menor de cinco hermanos y vive con sus padres, quienes trabajan y se hacen cargo de las necesidades de la familia. Todos los miembros juntos y los padres describen su relación de pareja como conflictiva y violenta. La mamá de Catalina, al enterarse de que su hija se autoagrede, es quien solicita la consulta psicológica y quien se muestra más preocupada y motivada al cambio.

Catalina presenta las características propias del SIB. Las conductas de autodaño fueron lesiones de corte moderado, en los brazos y la espalda, existiendo una secuencia inmediata entre el acto de herirse y la herida. La conducta se realizó siempre de manera privada y Catalina admitió haberse producido las lesiones, consciente del origen psicológico del daño. La conducta ha estado presente durante tres años de manera episódica.

Ella no ha asistido a otros procesos terapéuticos y hace tres meses que le ha contado a su madre que se produce lesiones, específicamente cortes en los brazos y parte superior de la espalda, comportamiento que se ha presentado durante tres años sin el conocimiento de su familia. Se debe aclarar que, por las características propias del SIB, el síntoma puede presentarse por largos periodos con total desconocimiento de la familia o las personas que conviven con quien se autoagrede.

\section{Proceso Terapéutico}

\section{Definición del problema}

La queja inicial está cargada de emociones de angustia y frustración por parte de Catalina y de preocupación y miedo por parte de la madre frente al SIB. La madre y la hija se muestran motivadas al cambio. Su 
primera queja deja ver que la conducta es motivo de dolor y que consideran necesario detenerla por su gravedad:

Mamá: "Lo que yo quiero es que usted le haga una evaluación a Catalina, porque lo que ella hace no está bien, ella se está cortando y, la verdad, yo pienso que esto es de psiquiatra, no sé qué más hacer y creo que ella necesita ayuda... yo sé que es por la relación que llevo con su padre".

Catalina: "Yo siento que esto me supera. Yo quiero dejar de hacerlo, pero, a veces, el dolor que siento es tan grande que esto lo alivia. Quiero dejar de hacer esto, pero no sé cómo".

Se co-construye el motivo de consulta a partir de las quejas iniciales, y por las características del SIB, el foco u objetivo terapéutico se mantiene en la conducta problema. La conducta de autodaño (con referencia a la queja) deja de explicarse como algo fuera de control o patológico y pasa a explicarse como una forma de comunicación que surge dentro de un sistema de interacción particular.

Se parte primero de entender cómo se explican Catalina y su madre (quienes asisten a consulta) el síntoma o SIB, y se busca junto con el cliente una manera distinta de calificar o connotar la autoagresión. Este proceso, como se ha mencionado anteriormente, se llama reformulación. En este caso el síntoma o conducta de autodaño deja de ser un comportamiento aislado para entrar a ser parte de una dinámica familiar y ya no se ve como una conducta sobre la cual no se tiene control, pasando a ser una conducta que le permite a Catalina comunicar algo a sus padres y miembros de su familia.

Estas explicaciones que tenemos de los síntomas permiten que las personas interactúen de diversas maneras, y aquí emergen las pautas o circuitos de interacción que los mantienen. En este caso, los circuitos interaccionales sobre los cuales se buscó, se describen a continuación. 


\section{Circuitos de Interacción}

\section{A. Primer Circuito}

En el nivel de la acción, los padres discuten, se gritan, se ignoran, se ofenden, usando palabras soeces, con la intención de manifestar el conflicto que existe en la pareja. Catalina se siente abrumada y angustiada. En consecuencia presenta el SIB, logrando, por una parte, calmar la angustia que ella califica como incontrolable y, por otra, detener el conflicto entre sus padres, ya que éstos dejan de discutir y centran su atención en las dificultades de Catalina.

En el nivel del significado se encuentran las creencias de los padres acerca de que su relación es mala (premisa I) y de que les es imposible controlarse cuando se sienten agredidos por su cónyuge (premisa II). Por otro lado, están las creencias de Catalina, consistentes en que haciéndose daño controla sus emociones de angustia (premisa III) y que sus padres tienen muchas dificultades y no merecen tener más problemas (premisa IV). La Figura 1 ejemplifica el circuito descrito y la posición del cliente.

Mamá: "Es mi culpa, por la relación que llevo con su padre y ellos la conocen (se refiere a sus hijos)... es una relación de mierda, él se construyó una especie de departamento al lado de nuestra casa, porque yo no soporto vivir con él".

Catalina: "Cuando siento angustia, realmente no me puedo controlar, esto me sobrepasa... Quisiera dejar de hacerlo, pero no se cómo".

Figura 1 Posición de Cliente Primer Circuito

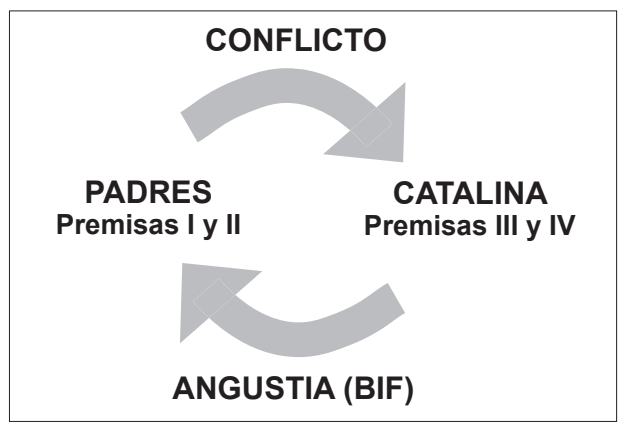




\section{B. Segundo Circuito}

Al detener el conflicto con su esposo, la madre centra su atención en Catalina. De esta manera el síntoma logra desviar la atención de la madre. Ésta se preocupa y ocupa toda la atención en ayudar a su hija, pasando el conflicto conyugal a un segundo plano y entrando en acción un segundo circuito establecido entre la madre y la hija. Este circuito presenta las siguientes características:

En el nivel de la acción, cuando se manifiesta el síntoma, la madre acosa a Catalina (pregunta insistentemente, la acompaña todo el día en casa, lee libros de auto ayuda) con la intención de protegerla y cuidarla, y en reacción a esta acción, Catalina se retira (se calla, se aísla, no habla) con el propósito de alejarse de la madre.

En el nivel del significado, se manifiesta la creencia de Catalina (premisa V) de que la madre no la deja tranquila y se preocupa demasiado, y la creencia de la madre (premisa VI) de que es necesario estar siempre con su hija y así evitar que se haga daño. En la Figura 2 se ejemplifica la posición del cliente en este segundo circuito.

Catalina: "Quisiera que mamá me deje tranquila. Desde que le conté dejó de trabajar".

Mamá: "Comencé a trabajar en casa, eso antes no lo hacía, pero me da miedo dejarla sola, asi que la vigilo y todos los días le pregunto si lo ha hecho o no".

Figura 2 Posición de Cliente Segundo Circuito

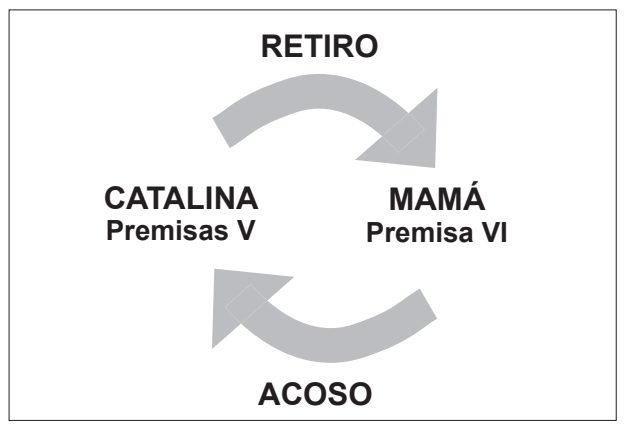




\section{Objetivo terapéutico}

El cambio está centrado en extinguir la manifestación del SIB, alterando el circuito de relaciones que lo mantiene. Así se reduce el riesgo de daño y se da a Catalina la sensación y certeza de que es algo que puede manejar. Posteriormente, se busca encontrar una manera menos dolorosa para que diga lo que siente, fortaleciendo el autoconcepto y los vínculos familiares.

La meta mínima está orientada, por una parte, a perturbar la pauta, y por otra, a invitar a Catalina a que se conecte con su emoción de angustia, haciéndola consciente cuando la escribe en un papel. El mensaje fue: “'por qué en lugar de escribir la angustia sobre tu cuerpo, no la escribes sobre un papel?”.

\section{Estrategias}

Las estrategias centrales fueron las reformulaciones y las prescripciones de comportamiento. En primer lugar, se entiende que reformular es ver un acontecimiento, situación o experiencia de una manera diferente desde otra perspectiva a la que se estaba usando, y al cambiar la forma de ver las cosas también cambia la manera en que actuamos y sentimos sobre estas cosas o experiencias.

La primera reformulación que se aplica en este caso clínico se presenta en la construcción del motivo de consulta, se cuestiona la premisa de Catalina de que el síntoma es algo inmanejable e incontrolable y este mismo síntoma pasa a ser un medio por el cual Catalina está comunicando algo. Se corta el cuerpo para expresar lo que no puede decir con palabras.

La segunda reformulación se aplica a la función que cumple el silencio de Catalina ante el SIB, puesto que ella le cuenta a su madre de los cortes en el cuerpo después de tres años de presentar la conducta de autoagresión. Se cuestiona la creencia de que el síntoma tiene la finalidad de dañar a Catalina y/o a sus padres y se introduce como una manera de proteger. La terapeuta lo presenta de la siguiente manera: 
Terapeuta: "Catalina, tú eres una niña tan respetuosa de lo que hacen tus papás y por eso aprendiste a escribir sobre tu cuerpo lo que no te atreves a decir con tus palabras, porque sabes que éstas pueden lastimar a las personas que más amas".

El objetivo de usar esta estrategia es cuestionar las creencias que acompañan a los consultantes, lograr un cambio a nivel cognitivo en la manera cómo se explica la realidad: Catalina se inmovilizaba, porque pensaba que la conducta de autoagredirse era incontrolable e inmodificable, y no había visto la relación de este síntoma con lo que pasaba con sus padres y las emociones de rabia y angustia que le producían las peleas entre ellos.

Pensar que la conducta de autoagredirse es una manera, pero no la única, de comunicar las emociones, le permitió a Catalina introducir y aceptar la idea de que puede hacer otra cosa para expresar lo que siente, sin agredirse ni poner en riesgo su salud.

Si la estrategia de la reformulación implica un cambio a nivel de las creencias, la segunda estrategia usada en las prescripciones de comportamiento permite un cambio a nivel de la conducta, ya que se busca, por medio de tareas concretas, alterar la pauta de interacción en la que se presenta la conducta problema. La primera prescripción de esta segunda estrategia busca alterar el circuito de interacción donde se manifiesta el síntoma, específicamente donde Catalina se autoagrede, y es presentada por la terapeuta de la siguiente manera:

Terapeuta: "Catalina, yo no te voy a decir que no lo hagas ni tampoco que lo hagas, por ahora necesitamos conocer mejor lo que te pasa y asi no nos apresuramos con lo que vamos a hacer. Por ello quiero que esta semana, cuando tengas ganas de cortarte, antes de hacerlo lo escribas. Lo importante es que lo hagas antes de cortarte, de esta manera podemos tener más claridad de lo que pasa". 
El objetivo principal es alterar la pauta en la que se presenta la conducta de autoagresión, confirmando la creencia de la madre (presentada en la queja) de que es necesario evaluar lo que pasa antes de tomar decisiones, postura que indica a los padres y a Catalina que el cambio se da poco a poco y de esta manera se reduce la ansiedad de ver prontos resultados. Por otro lado, se busca conjugar lo anterior con el mensaje de parte del terapeuta de que el proceso terapéutico es un camino en el cual están involucrados todos los miembros de la familia y no sólo la persona que manifiesta el síntoma, validando al mismo tiempo la angustia de Catalina y la manifestación de ésta.

Catalina: "La verdad quise hacer la tarea que usted me dejó... pero la verdad me dio mucha lata asi que dejé que todo pasara".

Una segunda prescripción de comportamiento, dentro de la segunda estrategia, se orienta a alterar el circuito de interacción que establece Catalina con sus padres y es presentada después de escuchar cómo se siente en relación con el conflicto de la pareja, y con lo que sus padres hacen ante eso. Se les pide a los padres que salgan de la consulta y que escriban una carta (y se la entreguen ahí mismo), diciéndole que ella no tiene que seguir siendo tan buena hija y callarse las cosas ni tratar de cuidarlos.

El objetivo de esta estrategia es incluir otra forma de comunicación en el sistema familiar. Catalina y sus padres hablan abiertamente de las emociones que los acompañan y de lo que esperan unos de otros, alterando así la manera de comunicación que ella había establecido con sus padres donde las autoagresiones tenían una función específica que es interrumpir el conflicto entre la pareja. La función de esta segunda prescripción se ve en la siguiente intervención de Catalina:

Catalina: "Las cosas en casa están mucho mejor, mi mamá por lo menos ya no me habla mal de mi papá. Claro que no entiendo por qué usted les hizo escribir esa carta, pero por lo menos mis papás ya no se gritan como antes". 


\section{Evaluación clínica final}

El caso se dio de alta por cumplimiento de objetivos y se sobrepasó la meta mínima pactada, que era la interrupción de la pauta y, en consecuencia, que Catalina dejase de dañarse. Después de cinco meses y dos semanas se conversó personalmente con ella y el cambio se había mantenido. Es de destacar que esa semana ${ }^{3}$ Catalina refirió que hace exactamente nueve meses que no se cortaba.

Catalina: "Mi mamá confía más en mí, ya no me anda presionando con que le cuente las cosas y eso me gusta... Las profesoras me están ayudando a pasar los ramos y bueno, si repito el año -ya lo hablé con mi mamá- no importa".

\section{Discusión}

La presente discusión se centrará en los aspectos de la intervención que se consideran más útiles y que permitieron el cambio terapéutico en un caso donde se presentaba el síntoma de autodaño o SIB. La aplicación del modelo comienza con la identificación de un problema. Si bien la existencia del síntoma es clara, éste se ubica dentro de un sistema y los miembros que lo conforman. En este caso, Catalina y su familia son quienes califican la autoagresión como una conducta indeseable, se muestran preocupados, desean modificarla y, en consecuencia, buscan ayuda ante intentos fallidos de solución.

El SIB es visto como una experiencia vital que comparten los miembros de la familia de Catalina y ante la cual todos se relacionan e interactúan, entretejiendo pautas y creencias que se mantienen, ya que están construidas desde su manera de ver, de sentir y de experimentar la realidad. $\mathrm{Al}$ aplicar el modelo se busca alterar el nivel de las creencias y el nivel de las conductas y, en consecuencia, generar un cambio, construyendo estrategias que responden a este objetivo. Lo que se persigue es lograr un cambio en la manera de ver la realidad y de explicar el síntoma, por lo que se aplica la estrategia de reformulación en co-construcción con Catalina, para ge-

\footnotetext{
Se hace referencia a la semana donde se realizó la evaluación del proceso.
} 
nerar un cambio de significado. Es decir, el SIB deja de ser una conducta enferma y dañina, pasando a ser una expresión de cuidado de Catalina para con sus padres. Ella prefiere hacerse daño en su cuerpo antes que hacerles daño a sus padres con lo que pueda llegar a decirles. La estrategia aplicada permite poner en duda dentro del sistema consultante la premisa o creencia de la madre de Catalina de que su hija está enferma. Al cambiar esta premisa cambia también la pauta de relación establecida por los miembros de la familia, especialmente los padres y la hija. Deja de ser una hija que no se controla, y pasa a ser una hija que protege con su silencio. "Las maniobras verbales de reestructuración, en efecto, han de suscitar dudas que ocupen el lugar de la habitual rigidez perceptivo-reactiva de los pacientes" (Nardone y Watzlawick., 1992, p. 91). Al cambiar la manera de ver una realidad cambia también la manera de reaccionar ante ella.

Para lograr modificar la pauta donde se presenta y es funcional el síntoma, se usan dos circuitos de interacción y esto nos permite realizar el diagnóstico. El primer circuito es el que se presenta entre Catalina y sus padres, donde se da una pauta simétrica de perpetuación del conflicto: en la medida que el conflicto conyugal aumenta, la angustia de Catalina aumenta.

Es en la escalada de la discusión conyugal, el segundo circuito, donde el SIB aparece cumpliendo las siguientes funciones: a) En primer lugar, el SIB le permite a Catalina autorregularse emocionalmente, disminuyendo y controlando la angustia que le produce el conflicto entre sus padres. B) En segundo lugar, el SIB desvía y distrae la atención de la madre de los problemas con su esposo. Se debe aclarar que esta segunda función del síntoma se presenta durante el periodo ( 3 meses) en el cual se conoce en la familia la presencia del SIB y se busca terapia psicológica.

Las estrategias se orientan específicamente al síntoma, la emoción que lo acompaña y la manera de comunicarla de una forma menos riesgosa que el autodańo. Se le prescribe a Catalina introducir una nueva conducta en la secuencia de interacción buscando perturbar la pauta. En el momento en que la sensación de angustia aumente, Catalina debe escribir en un papel sobre esa angustia y cómo la siente su cuerpo en ese 
momento. Para ello se usa la metáfora de que los cortes son una manera de escribir en el cuerpo lo que no se puede decir con las palabras, por lo tanto, la prescripción no se presenta a Catalina como una manera de interrumpir la autoagresión, sino como una manera de que Catalina diga lo que siente y piensa, reconociendo lo que ella comunica como valioso e importante. Catalina no es la portadora de un síntoma, sino que es una joven que trata de decir lo que siente por medio de una manifestación conductual muy concreta, a saber, los cortes que ella misma se produce en el cuerpo.

De esta manera se valida la necesidad de Catalina de comunicar y controlar la angustia. Lo que se pone en duda es el cómo comunicarla y controlarla, de la piel al papel, aspecto que se considera fundamental para lograr el cumplimiento de los objetivos pautados con el sistema consultante, ya que se respeta la construcción de mundo del paciente y las intervenciones se acoplan al estilo particular de Catalina.

Es importante resaltar que cuando se detienen las peleas de los padres (al presentarse el SIB) emerge el segundo circuito diagnóstico, el de Catalina con su madre, donde se da una pauta complementaria de acoso-retiro. Catalina presenta el SIB y su madre le prodiga todo el cuidado. En este circuito, a diferencia del primero, Catalina deja de cuidar, para ser cuidada. Ella produce un síntoma como el autodaño que logra provocar el ruido suficiente para detener el conflicto conyugal, se da el SIB y esto produce que sea perseguida y controlada. Corta con sus cortes en el cuerpo lo que la perturba, en este caso las peleas de sus padres.

En este proceso, las prescripciones destinadas a modificar este segundo circuito son las que más impacto tienen en Catalina, lo que es comprensible si se tiene en cuenta que ella busca independencia y espacio. Por lo tanto, poder conseguirlo la motiva al cambio y a participar activamente en el proceso.

Se considera que fue significativo para la consultante el espacio que se le dio dentro del proceso, pues si bien el foco se mantuvo en los circuitos de relaciones, Catalina es validada en sus necesidades y expectativas de acuerdo con su edad. Esto le permite encontrar los recursos para modificar 
esta conducta que evidentemente no sólo es peligrosa, sino también, dolorosa para ella y para su familia.

Una de las fortalezas de este enfoque es que da directrices claras en los abordajes de este tipo de temáticas, que muchas veces tienden a ser derivados por terapeutas jóvenes por no tener, como es el caso de la terapeuta, una experiencia previa que avalara su intervención.

Finalmente, aquí se presenta un caso clínico con características particulares, por lo tanto, no se busca generalizar la intervención realizada ni se presenta como una única alternativa, pero se muestran aspectos del proceso clínico que explican cómo el modelo del MRI y la aplicación de las estrategias permitieron un cambio. Finalmente, el SIB es un síntoma ante el cual quedan muchos interrogantes e investigaciones por realizar y ésta es una manera de contribuir al trabajo que muchos realizan para hacer frente a un síntoma que, aunque se caracteriza por el silencio de quien se auto arremete, cada vez afecta a más jóvenes en Chile y en el mundo.

\section{Referencias bibliográficas}

AMERican pSyChiatric association. (2000). Diagnostic and statistical manual of mental disorders. Washington D.C.: APA.

Anderson, H. (1999). Conversación, lenguaje y posibilidades. Buenos Aires: Amorrortu.

Casabianca, R. y Hirsch, H. (1994). Cómo equivocarse menos en terapia. Argentina: Universidad Nacional del Litoral.

Ciberio, M. Hablemos el mismo idioma. Hablar el lenguaje del paciente. Extraído el 5 de septiembre de 2008 desde

http://www.escuelasistemica.com.ar/publicaciones/articulos/10.pdf

Claes, L. y Vandereycken, W. (2007). Self-injurious behavior: Differential diagnosis and functional differentiation. Comprehensive Psychiatry Review, 48, 137 - 144.

Feixas, G. y Villegas, M. (2004). Constructivismo en psicoterapia. Bilbao: Desclée de Brouwer.

Fisch, R. y Schlanger, K. (2002). Cambiando lo incambiable. Barcelona: Herder.

Fisch, R. Segal, L. y Weakland, J. (1988). La táctica del cambio: Cómo abreviar la terapia. Barcelona: Herder. 
Graff, H. y Mallin, R. (1967). The syndrome of the wrist cutter. American Journal of Psychiatry, 124, 36-42.

Kuonsky, D. (2007). The functions of deliberate self-injury: A review of the evidence. Clinical Psychology Review, 27, 226 - 239.

Nardone, G. y Watzlawick, P. (1992). El arte del cambio. Barcelona: Herder.

Procter, H. (1987). A personal construct approach to family therapy and systems intervention. En E. J. Button (1985), Personal construct theory and mental health: Theory, Research, and Practice (pp. 327 350). Michigan: Brookline Books.

Neimeyer, R. y Mahoney, P. (1995). Constructivismo en Psicoterapia. Buenos Aires: Paidós.

Purington, A. y Whitlock, J. (2004). Research facts and findings: Self injury facts and for youth upstate center for excellence. Publication of Cornell University, U. of Rochester and New York State Center for School Safety.

WatzlaWick, P., Beavin, B. Jackson, D. (1997). Teoría de la comunicación humana: interacciones patologías y paradojas. Barcelona: Herder.

Whitlock, J., Eckenrode, J y Silverman, D. (2006). Self injurious behavior in a college population. Pediatrics. 6, $117-133$.

Whitlock, J. y Knox, K. (2007). The relationship between suicide and self injury in young adult population, Archives of Pediatrics and Adolescent Medicine, 7, 161 - 183.

Fecha de Recepción de artículo: 16 de junio 2008

Fecha de Aceptación de artículo: 20 de agosto 2008 
